\title{
Fatores de risco em adultos jovens para o desenvolvimento de doenças cardiovasculares: o que a literatura mostra?
}

\author{
Risk factors in young adults for the development of cardiovascular disease: what does the literature
} show?

Factores de riesgo en adultos jóvenes para el desarrollo de enfermedades cardiovasculares: ¿qué muestra la literatura?

\author{
João Daniel de Souza Menezes \\ ORCID: https://orcid.org/0000-0001-8767-7556 \\ Faculdade de Medicina de São José do Rio Preto, Brasil \\ Liga de Cardiologia - LICARDIO, Brasil \\ E-mail: joao.menezes@edu.famerp.br \\ Andressa Muzzo de Souza \\ ORCID: https://orcid.org/0000-0002-5957-5891 \\ Faculdade de Medicina de São José do Rio Preto, Brasil \\ Liga de Cardiologia - LICARDIO, Brasil \\ E-mail: andressamuzzo59@gmail.com \\ Viviane Thais de Oliveira Fraga \\ ORCID: https://orcid.org/0000-0001-5899-4376 \\ Faculdade de Medicina de São José do Rio Preto, Brasil \\ Liga de Cardiologia - LICARDIO, Brasil \\ E-mail: viviane.fraga@edu.famerp.br \\ Moacir Fernandes de Godoy \\ ORCID: https://orcid.org/0000-0001-8390-0933 \\ Faculdade de Medicina de São José do Rio Preto, Brasil \\ Liga de Cardiologia - LICARDIO, Brasil \\ E-mail:mf60204@gmail.com
}

\begin{abstract}
Resumo
Introdução: As doenças cardiovasculares representam um grande ônus para a saúde pública, são a principal causa de morte no mundo e têm gerado perda da qualidade de vida, limitações nas atividades de trabalho e lazer, além de impactos econômicos às famílias e à sociedade em geral. Embora se reconheça que as doenças cardiovasculares e a hipertensão arterial sejam mais comumente percebidas em indivíduos em idade adulta, a manifestação destas doenças é resultante da interação de fatores de risco adquiridos ainda em idade jovem. Objetivo: O presente estudo teve como objetivo avaliar os fatores de risco associados ao desenvolvimento de doenças cardiovasculares em adultos jovens. Metodologia: Realizou-se uma revisão de estudos sobre os fatores de risco para hipertensão e cardiopatias em adultos jovens nas bases de dados PubMed/Medline, Lilacs e Scielo. Não houve restrição quanto ao idioma utilizado nas publicações. A pesquisa foi realizada em abril de 2021, utilizando-se os descritores "fatores de risco", "doenças cardiovasculares", "adultos jovens" e "hipertensão". Resultados: Com base nos estudos abordados, o risco de doenças cardiovasculares e hipertensão arterial pode iniciar já nas primeiras décadas de vida, haja vista que os adolescentes e indivíduos em idade jovem compõem um importante grupo vulnerável às situações de exposição aos fatores de risco para o desenvolvimento de eventos cardiovasculares maléficos, com destaque para os relacionados ao estilo de vida como sedentarismo, alcoolismo, tabagismo, má adesão à atividade física regular e aumento de índice de massa corpórea por uma alimentação baseada em alta densidade calórica. Conclusões: Os estudos ressaltam a importância de se delimitar os fatores de risco no início da vida associados ao desenvolvimento de doenças cardiovasculares e hipertensão arterial que possam focalizar e informar medidas preventivas ou facilitar a adoção de abordagens que promovam comportamentos e atitudes saudáveis ao longo da vida.
\end{abstract}

Palavras-chave: Fatores de risco; Hipertensão; Doenças cardiovasculares.

\begin{abstract}
Introduction: Cardiovascular diseases represent a major burden to public health, are the leading cause of death worldwide and have generated loss of quality of life, limitations in work and leisure activities, in addition to economic impacts to families and society in general. Although it is recognized that cardiovascular diseases and hypertension are more commonly seen in individuals in adulthood, the manifestation of these diseases results from the interaction of risk factors acquired at a young age. Objective: The present study aimed to evaluate the risk factors associated with the development of cardiovascular diseases in young adults. Methodology: A review of studies on risk factors for hypertension and heart disease in young adults was conducted
\end{abstract}


in the PubMed/Medline, Lilacs and Scielo databases. There was no restriction as to the language used in the publications. The search was conducted in April 2021, using the descriptors "risk factors," "cardiovascular diseases," "young adults," and "hypertension." Results: Based on the studies addressed, the risk of cardiovascular diseases and hypertension may begin as early as the first decades of life, given that adolescents and individuals at a young age make up an important vulnerable group to situations of exposure to risk factors for the development of harmful cardiovascular events, with emphasis on those related to lifestyle such as sedentarism, alcoholism, smoking, poor adherence to regular physical activity and increased body mass index by a diet based on high caloric density. Conclusions: The studies highlight the importance of delineating the risk factors early in life associated with the development of cardiovascular disease and hypertension that can focus and inform preventive measures or facilitate the adoption of approaches that promote healthy behaviors and attitudes throughout life.

Keywords: Risk factors; Hypertension; Cardiovascular diseases.

\section{Resumen}

Introducción: Las enfermedades cardiovasculares representan una gran carga para la salud pública, son la principal causa de muerte en el mundo y provocan pérdida de calidad de vida, limitaciones en las actividades laborales y de ocio, además de impactos económicos para las familias y la sociedad en general. Aunque se reconoce que las enfermedades cardiovasculares y la hipertensión se perciben más comúnmente en los individuos en la edad adulta, la manifestación de estas enfermedades resulta de la interacción de factores de riesgo adquiridos a una edad temprana. Objetivo: El presente estudio tuvo como objetivo evaluar los factores de riesgo asociados al desarrollo de enfermedades cardiovasculares en adultos jóvenes. Metodología: Se realizó una revisión de los estudios sobre los factores de riesgo de la hipertensión y la cardiopatía en adultos jóvenes en las bases de datos PubMed/Medline, Lilacs y Scielo. No había ninguna restricción en cuanto a la lengua utilizada en las publicaciones. La búsqueda se realizó en abril de 2021, utilizando los descriptores "risk factors", "cardiovascular diseases", "young adults" y "hypertension". Resultados: Con base en los estudios abordados, el riesgo de enfermedades cardiovasculares e hipertensión puede iniciarse ya en las primeras décadas de la vida, dado que los adolescentes e individuos en edad temprana conforman un importante grupo vulnerable a situaciones de exposición a factores de riesgo para el desarrollo de eventos cardiovasculares nocivos, destacando los relacionados con el estilo de vida como el sedentarismo, el alcoholismo, el tabaquismo, la poca adherencia a la actividad física regular y el aumento del índice de masa corporal por una dieta basada en una alta densidad calórica. Conclusiones: Los estudios destacan la importancia de delimitar los factores de riesgo en las primeras etapas de la vida asociados al desarrollo de enfermedades cardiovasculares e hipertensión que pueden enfocar e informar las medidas preventivas o facilitar la adopción de enfoques que promuevan comportamientos y actitudes saludables a lo largo de la vida.

Palabras clave: Factores de riesgo; Hipertensión; Enfermedades cardiovasculares.

\section{Introdução}

As doenças cardiovasculares representam um grande ônus para a saúde pública, são a principal causa de morte no mundo e têm gerado perda da qualidade de vida, limitações nas atividades de trabalho e lazer, além de impactos econômicos para as famílias e sociedade em geral (Ezeudu et. al., 2018 \& Li, 2020). A etiologia multifatorial das doenças cardiovasculares é amplamente conhecida, os fatores de risco são classificados em duas modalidades: (1) aspectos não modificáveis tais como hereditariedade, idade, sexo e (2) fatores de risco passíveis de intervenção e modificação como hipertensão arterial sistêmica, colesterol aumentado, tabagismo, sedentarismo, obesidade, diabetes mellitus e fatores psicossociais (Wittkopf et. al., 2016 \& Chrysaidou, 2020).

As doenças cardiovasculares são, na atualidade, responsáveis por aproximadamente $40 \%$ da mortalidade no mundo (Yusuf et. al., 2001 \& Jakab, 2020). Tal fato se alicerça na premissa de que a prevalência dessa enfermidade vem aumentando (Hajjar, 2003 \& Genovesi, 2020) bem como na perspectiva de que níveis pressóricos elevados têm forte impacto no aumento de risco para doenças cardiovasculares (Lewington, 2002 \& Guzman-Limon et. al., 2019) e seu diagnóstico em fase precoce vem apontando como importante estratégia de saúde pública (Wang \& Necheles, 2008; Siddiqui \& Malatesta-Muncher, 2020).

O aumento na prevalência de hipertensão arterial sistêmica (HAS) parece não se restringir à população adulta, a literatura advoga que tal quadro atinge também adolescentes. Diante do presente contexto transparece a necessidade do diagnóstico da HAS em fases precoces da vida do sujeito.

Embora se reconheça que as doenças cardiovasculares e a HAS sejam mais comumente percebidas em indivíduos em idade adulta, a manifestação destas doenças é resultante da interação de fatores de risco adquiridos ainda em idade jovem. 


\section{Metodologia}

Realizou-se uma revisão de estudos sobre os fatores de risco para hipertensão e cardiopatias em adultos jovens nas bases de dados PubMed/Medline (National Library of Medicine), Lilacs (Literatura Latino-Americana e do Caribe em Ciências da Saúde) e Scielo (Scientific Electronic Library Online) (Estrela, 2018).

\section{Estratégias de Busca}

As buscas virtuais foram realizadas nas bases de dados utilizando os operadores booleanos. Buscou-se por dados mais recentes através das seguintes estratégias: "risk factors for heart disease AND young adults", "biopsychosocial profile AND heart disease", "biopsychosocial profile AND hypertension AND young adult". As pesquisas foram realizadas em abril de 2021. Não houve restrição quanto ao idioma utilizado nas publicações.

\section{Critérios de Inclusão e exclusão dos estudos}

Os estudos foram elegíveis considerando os seguintes aspectos: 1) fossem artigos originais e 2) avaliassem as relações entre hipertensão, cardiopatias e adultos jovens. O principal critério de exclusão foi: artigos que não abordassem a população de adultos jovens. Inicialmente, os títulos e resumos dos artigos foram analisados a fim de verificar se atendiam aos critérios de inclusão definidos e/ou se apresentavam critério de exclusão (Estrela, 2018 \& Sousa, 2017).

\section{Busca na literatura}

Dos 34 artigos selecionados pelo título, 19 foram excluídos após a leitura do resumo pois não atendiam aos critérios de inclusão e 15 artigos foram selecionados para a leitura do texto completo. Após os textos completos serem analisados por três avaliadores, 5 artigos foram excluídos por não abordarem, exclusivamente, as correlações entre hipertensão, cardiopatias e adultos jovens ou por terem abordado outras faixas etárias.

\section{Resultados e Discussão}

Após a leitura e desenvolvimento do método de seleção de artigos, elaborou-se uma tabela (Quadro 1) para melhor visualização e compreensão acerca dos artigos selecionados para compor esta revisão de literatura. Os artigos selecionados correspondem ao nível de evidência VI (Melnyk, 2005). 
Quadro 1. Distribuição dos artigos incluídos na revisão conforme autor/ ano, palavra-chave, metodologia, amostra e nível de evidência. São José do Rio Preto - SP; 2021.

\begin{tabular}{|c|c|c|c|c|}
\hline Autor/ano & Palavra-chave & Metodologia & Amostra & $\begin{array}{ll}\begin{array}{l}\text { Nível } \\
\text { evidência }\end{array} & \text { de }\end{array}$ \\
\hline Soares et. al., 2020; & $\begin{array}{l}\text { Hipertensão arterial sistêmica, } \\
\text { adolescentes, fatores de risco. }\end{array}$ & $\begin{array}{l}\text { Pesquisa de levantamento } \\
\text { observacional e de campo, } \\
\text { transversal. }\end{array}$ & 62 participantes & Nível VI \\
\hline Silva et. al., 2020; & $\begin{array}{l}\text { Fatores de risco. Doenças } \\
\text { cardiovasculares. Adulto jovem. } \\
\text { Renda. }\end{array}$ & Estudo seccional & $\begin{array}{l}2.063 \text { indivíduos aos } \\
23 / 25 \text { anos }\end{array}$ & Nível VI \\
\hline $\begin{array}{l}\text { Avelino et. al., } \\
\text { 2020; }\end{array}$ & $\begin{array}{l}\text { Doença Cardiovascular, Fatores } \\
\text { de Risco, Sedentarismo. }\end{array}$ & $\begin{array}{l}\text { Estudo de caráter transversal, } \\
\text { descritivo, com abordagem } \\
\text { quantitativa. }\end{array}$ & $\begin{array}{l}30 \text { indivíduos na faixa } \\
\text { etária } \\
\text { entre } 20 \text { e } 24 \text { anos. }\end{array}$ & Nível VI \\
\hline $\begin{array}{l}\text { Cassiano et. al., } \\
\text { 2019; }\end{array}$ & $\begin{array}{lr}\text { Antropometria, } & \text { Estado } \\
\text { Nutricional, } & \text { Hipertensão } \\
\text { Arterial. } & \end{array}$ & $\begin{array}{l}\text { Estudo quantitativo, do tipo } \\
\text { transversal. }\end{array}$ & $\begin{array}{l}86 \text { indivíduos com } \\
\text { idade média de 19,0 } \pm \\
0,97 \text { anos. }\end{array}$ & Nível VI \\
\hline Santos et. al., 2018; & $\begin{array}{l}\text { Hipertensão. Fatores de Risco. } \\
\text { Prevalência. }\end{array}$ & $\begin{array}{l}\text { Estudo de } \\
\text { corte transversal descritivo. }\end{array}$ & $\begin{array}{l}60 \text { acadêmicos, na } \\
\text { faixa etária entre } 18 \text { a } \\
30 \text { anos. }\end{array}$ & Nível VI \\
\hline Pelazza et. al., 2019; & $\begin{array}{lr}\text { Adolescentes; } & \text { Ansiedade; } \\
\text { Estudantes; } & \text { Hipertensão; } \\
\text { Ensino . } & \end{array}$ & $\begin{array}{l}\text { Estudo quantitativo, transversal e } \\
\text { descritivo. }\end{array}$ & $\begin{array}{l}96 \text { estudantes com } \\
\text { média de idade de } 18,2 \\
\text { anos. }\end{array}$ & Nível VI \\
\hline $\begin{array}{l}\text { Bergmann et. al., } \\
\text { 2018; }\end{array}$ & $\begin{array}{l}\text { Obesidade; } \\
\text { Hipertensão; Colesterol; } \\
\text { saudáveis; Adolescentes. }\end{array}$ & Cross-sectional study. & $\begin{array}{l}1.045 \text { adolescentes, de } \\
11 \text { a } 17 \text { anos de idade. }\end{array}$ & Nível VI \\
\hline Bozza et. al., 2016; & $\begin{array}{l}\text { Pressão Arterial; Hipertensão / } \\
\text { genética; Circunferência da } \\
\text { Cintura; Fatores de Risco; } \\
\text { Adolescente. }\end{array}$ & $\begin{array}{l}\text { Estudo de delineamento } \\
\text { transversal }\end{array}$ & $\begin{array}{l}1.242 \text { adolescentes de } \\
11 \text { a } 17 \text { anos de idade. }\end{array}$ & Nível VI \\
\hline Costa et. al., 2017; & $\begin{array}{l}\text { atividade física; comportamento } \\
\text { sedentário; doenças } \\
\text { cardiovasculares; adolescentes. }\end{array}$ & Estudo transversal & $\begin{array}{l}576 \text { adolescentes de } 15 \\
\text { a } 19 \text { anos. }\end{array}$ & Nível VI \\
\hline Jeon et. al., 2020; & $\begin{array}{l}\text { Bidirectional association; blood } \\
\text { pressure; } \\
\text { cohort study; } \\
\text { depressive } \\
\text { symptoms; } \\
\text { hypotension }\end{array}$ & Prospective cohort study & 276.244 adults & Nível VI \\
\hline
\end{tabular}

O desenvolvimento adolescente compreende muitas mudanças com alterações psicossociais, biológicas, alteração da estima e em suas relações e atividades diárias, este público pode desenvolver em seu dia a dia atividades acadêmicas e de recreação, participação essas que antes não desenvolviam, isso se torna um possível fator de estresse. (Pelazza et. al., 2019 \& Costa et. al., 2017).

Muitos adolescentes estão envoltos a vulnerabilidades sociais e financeiras, em decorrência de muitos fatores, sendo que nessa fase muitos transtornos mentais ficam evidentes, como a depressão, crises de ansiedade e algumas patologias mais específicas; é notório que em muitos casos por falta de apoio ou educação em saúde, muitos adolescentes acabam compreendendo a definição de saúde de forma equivocada, permeando apenas a ausência de doença, compreensão essa limita, uma vez que a Organização Mundial de Saúde (OMS) define saúde como um bem estar biopsicossocial, e não apenas a ausência de patologias 
(Pelazza et. al., 2019 \& Silva et. al., 2020).

Quando pensado nos riscos cardiovasculares presentes na adolescência pode-se identificar alguns sinais e sintomas que podem estar presentes, como insônia, tremor, elevação dos valores pressóricos, ganho de peso, ansiedade exacerbada, dores no peito (angina), alimentação desequilibrada, pouca ingesta hídrica diária, sedentarismo, entre outros fatores que corroboram para um risco elevado de doenças cardiovasculares (Pelazza et. al., 2019).

Dessa forma foram elencados três subitens para melhor compreensão acerca deste estudo, sendo eles: fatores de risco, dados antropométricos versus doenças cardiovasculares e a importância da inserção da atividade física no dia a dia.

\section{Fatores de risco}

Como já mencionado, grande parte da população vive em situação de extrema vulnerabilidade social, sendo mais evidente durante o processo de pandemia do vírus SARS-CoV-2, muito destes fatores contribuem para educação em saúde precária, sem o nível de atenção adequada, como o consumo de alimentos industrializados e ultraprocessados, consumo de hidricos com alta porcentagem de sódio e açúcares, como os refrigerantes, isso pode ser explicado por muitos motivos, pois esses produtos são de baixo custo e fácil consumo. $\mathrm{O}$ aumento considerável no peso corporal, é um dos fatores de risco para o desenvolvimento de alterações pressóricas, deve-se seguir as recomendações das organizações em saúde referente ao peso ideal para faixa etária e sexo (Jeon et. al., 2020).

Além disso, já é consenso nas classes de saúde que o ganho de peso e o risco para desenvolvimento de alterações pressóricas estão intimamente ligados, sendo um dos marcadores de comprometimento da saúde cardiovascular, a HAS é responsável por 54\% das doenças cerebrovasculares e 47\% das doenças isquêmicas do coração (Bozza et. al., 2016).

Com o avanço da tecnologia e bens de consumo, fica necessário a utilização de ferramentas que não cobrem tanto tempo para seu desenvolvimento, com isso tem-se a menor adesão às práticas esportivas e recreativas em geral, muitos adolescentes estão deixando ou optando por não realizar exercícios físicos por muitos fatores, um deles é a falta de tempo para realização, já que muitos necessitam de vínculo empregatício para o sustento familiar. A falta de adesão ao exercício físico corrobora inversamente para a saúde cardíaca, o sedentarismo além de colaborar para piora da função cardiovascular, também oferece risco para outras manifestações patológicas tanto físicas como mentais (Soares et.al., 2020).

Em função disso, outra prática muito comum na população adulto jovem é o tabagismo, em parte da população mais vulnerável e com menor acesso aos recursos em saúde e educação, na qual a falta de apoio e suporte familiar faz com que muitos adolescentes se utilizem do tabagismo como recurso "terapêutico". Ao analisar o uso de cigarros, uso de drogas ilícitas e/ou lícitas, podemos notar a variedade de consequências observadas após a utilização dos mesmos, e uma das consequências comuns entre elas é a piora da função cardiopulmonar, podendo gerar além de alterações estruturais ou morfológicas, como DPOC doença pulmonar obstrutiva crônica e outras; até o surgimento de alterações psicossociais, como depressão e ansiedade, o que facilita no comprometimento em sociedade deste indivíduo (Costa et. al., 2017)

O risco para o desenvolvimento cardiovascular pode ser exacerbado durante a fase de adulto jovem, já que é a população em que se começa a oferecer o direito de escolha legal, e quando se há lacunas na educação pode se tornar prejuízo durante a vida adulta, um exemplo é o consumo de álcool, que em geral, começa durante a fase adulto jovem e pode percorrer por muitos anos de forma descontrolada, sem limites, gerando prejuízo em segmentos da vida, como na saúde, social e familiar. O consumo de álcool, favorece muito o desenvolvimento de doenças cardiovasculares, uma vez que se tem uma relação a nível cérebro vascular; é evidente que quando a sociedade deixa de orientar e ser um facilitador na educação dos adultos jovens, teremos alterações a nível de toda comunidade (Silva et. al., 2020).

Dessa forma, é possível identificar que quando há lacunas em atendimento e na educação da população desde a infância, a sociedade terá consequências em diversas perspectivas; é dever da comunidade ser difusora de conhecimento e é dever do 
estado oferecer saúde de qualidade e que permeia todo processo saúde doença, atuando desde prevenção e promoção até reabilitação em saúde, é notório a função social que o profissional de saúde desempenha uma vez que possui a domínio em relação a meios de identificação precoce de prejuízo em saúde (Silva et. al., 2020).

\section{Dados antropométricos versus doença cardiovascular}

Pensando no desenvolvimento do adolescente e ganho de peso, este um importante sinal de agravo em saúde, já que pode condicionar a situação de doença crônica não transmissível, como a hipertensão arterial sistêmica, em muitos casos essa elevação da PA está relacionada às condições socioeconômicas, bem como ao acesso aos serviços em saúde, como unidades básicas, acesso a exames laboratoriais e acompanhamento por profissionais de saúde (Cassiano et. al., 2019).

Assim, ao analisar um paciente com ganho de peso acima dos níveis estabelecidos em relação a altura, sexo e idade, devemos estar atentos aos sinais de gravidades, como níveis pressóricos elevados, insônia, diabetes mellitos, hipercolesterolemia, hipertrigliceridemia, e outros sinais e sintomas que podem estar presentes e representar gravidade a longo prazo.

A prevalência de HAS está relacionada às condições biopsicossociais, como tabagismo, obesidade, etilismo, sedentarismo, como já mencionados, e segundo a OPAS (Organização Pan Americana de Saúde), estima-se que 600 milhões de pessoas são hipertensas crônicas em todo o mundo, representando 13\% dos óbitos, como no caso do Brasil (Organização Panamericana Da Saúde, 2003).

Com isso, as políticas em saúde tem elaborados estratégias para melhor identificação e acesso a população com excesso de peso, para promoção em saúde, realizada por profissionais capacitados a identificar os fatores de risco presente para o desenvolvimento de doenças crônicas não transmissíveis, assim é necessário maior educação em saúde, para que as informações pertinentes aos fatores modificáveis e não modificáveis, bem como recursos para melhora da qualidade de vida estejam disponíveis para toda população (Bozza et. al., 2016).

\section{Importância da inserção da atividade física no dia a dia}

Nas fases de adolescência e início da adultez os indivíduos adquirem comportamentos que poderão resultar em efeitos deletérios à saúde, fato que fundamenta a necessidade de intervenções, por meio da promoção da educação em saúde, com vistas a reduzir as chances de desenvolverem algum evento de natureza cardiovascular durante a vida adulta. Tais intervenções são comumente associadas a mudanças no estilo de vida, representadas por incorporação de hábitos alimentares saudáveis e realização de atividade física regular (Avelino et. al., 2020).

Com isso, é sabido ser um processo demorado e que empenhe dedicação para sua conclusão, muitas alterações em saúde podem ser recuperadas ou amenizadas com o uso de práticas diárias de exercício físico, como caminhada, passeios de bicicleta, coisas fora das atividades desenvolvidas durante o dia a dia, como ir à escola ou ao trabalho, é necessário compreender ser um processo demorado, pois para o desenvolvimento das práticas é fundamental o condicionamento físico (Bergmann et. al., 2018).

Muitos esportes podem ser considerados úteis para suprir a necessidade do paciente sedentário, destes grande maioria podem ser realizados em grupos, o que auxilia neste processo, uma vez que desenvolver algo entre indivíduos possibilita a comunicação, quebra da ansiedade e da barreira social, sendo um importante mecanismo de inserção a nível social e comunitário (Bergmann et. al., 2018 \& Avelino et. al., 2020).

Para melhores resultados é necessário adaptações também em relação à alimentação, o que contribui positivamente para o processo saúde-doença. É notório que o acompanhamento de profissional adequado se faz necessário, consultas com nutricionistas tornam possível a identificação de maneiras de suprir a necessidade individual em relação aos nutrientes e fatores energéticos disponíveis na dieta (Santos et. al., 2018). 


\section{Conclusão}

Considerando os grandes prejuízos humanos e financeiros promovidos pelas doenças cardiovasculares, continua sendo imprescindível encontrar fatores de risco no início da vida associados ao desenvolvimento de doenças cardiovasculares que possam focalizar e informar medidas preventivas ou facilitar a adoção de abordagens que promovam comportamentos e atitudes saudáveis ao longo da vida.

Adolescentes e indivíduos em idade jovem, em geral, compõem um importante grupo vulnerável às situações de exposição a fatores de risco para o desenvolvimento de eventos cardiovasculares, com destaque para os relacionados ao estilo de vida. Assim, há necessidade da identificação e controle dos fatores de risco para que estratégias de promoção de saúde sejam desenvolvidas e que minimizem o risco do desenvolvimento de cardiopatias e hipertensão arterial. Para tanto, indivíduos expostos aos fatores de risco necessitam de uma mudança nos hábitos de vida como diminuição da ingestão de alimentos de alta densidade calórica, prática de atividade física regular, e cessação do tabagismo e alcoolismo. Dessa forma como sugestão para artigos futuros se tem a realização de estudos em campo analisando características laboratoriais dos adultos jovens com risco para doenças cardiovasculares.

\section{Referências}

Avelino, E. B., de Abreu Morais, P. S., da Costa Santos, A. C. B., Bovi, A. C. N., Paz, N. H., da Silva Santos, A. L., \&amp; de Morais Lima, J. H. (2020). Fatores de risco para doença cardiovascular em adultos jovens sedentários. Brazilian Journal of Development, 6(8), 58843-58854.

Bergmann, G. G., Tassitano, R. M., de Araújo Bergmann, M. L., Tenório, M. C. M., \& Mota, J. (2018). Screen time, physical activity and cardiovascular risk factors in adolescents. Revista Brasileira de Atividade Física; Saúde, 23, 1-12.

Bozza, R., Campos, W. D., Barbosa, V. C., Stabelini, A., Silva, M. P. D., \& Maziero, R. S. B. (2016). Pressão Arterial Alterada em Adolescentes de Curitiba: Prevalência e Fatores Associados. Arquivos Brasileiros de Cardiologia, 106, 411-418.

Cassiano, M. H., Luz, A. B. S., Bezerra, M. S., Barbosa, S. S., da Silva, H. T. D., \& de Souza Araújo, D. F. (2019). Correlação entre os índices antropométricos e pressão arterial de adolescentes e adultos jovens em um município do nordeste brasileiro. Revista Ciência Plural, 5(2), 49-67.

Chrysaidou, K., Chainoglou, A., Karava, V., Dotis, J., Printza, N., \& Stabouli, S. (2020). Secondary hypertension in children and adolescents: novel insights. Current hypertension reviews, 16(1), 37-44.

Corrêa-Neto, V. G., Sperandei, S., Silva, L. A. I., Maranhão-Neto, G. D. A., \& Palma, A. (2014). Hipertensão arterial em adolescentes do Rio de Janeiro: prevalência e associação com atividade física e obesidade. Ciência \& Saúde Coletiva, 19, 1699-1708.

Costa, I. F. A. F. D., Medeiros, C. C. M., Costa, F. D. A. F. D., Farias, C. R. L. D., Souza, D. R., Adriano, W. S., \& Carvalho, D. F. (2017). Adolescentes: comportamento e risco cardiovascular. Jornal Vascular Brasileiro, 16, 205-213.

Estrela, C. (2018). Metodologia Científica: Ciência, Ensino, Pesquisa. Editora Artes Médicas.

Ezeudu, C. E., Chukwuka, J. O., Ebenebe, J. C., Igwe, W. C., \& Egbuonu, I. (2018). Hypertension and prehypertension among adolescents attending secondary schools in urban area of South-East, Nigeria. Pan African Medical Journal, 31(1).

Genovesi, S., Parati, G., Giussani, M., Bona, G., Fava, C., Maffeis, C., \& Giordano, U. (2020). How to apply European and American Guidelines on high blood pressure in children and adolescents. A position paper endorsed by the Italian Society of Hypertension and the Italian Society of Pediatrics. High Blood Pressure \& Cardiovascular Prevention, 27(3), 183-193.

Guzman-Limon, M., \& Samuels, J. (2019). Pediatric hypertension: diagnosis, evaluation, and treatment. Pediatric Clinics, 66(1), 45-57.

Hajjar, I., \& Kotchen, T. A. (2003). Tendências na prevalência, conscientização, tratamento e controle da hipertensão nos Estados Unidos, 1988-2000. Jama, 290 (2), 199-206.

Jakab, A. E., Hidvégi, E. V., Illyés, M., Cziráki, A., Kalmár, T., Maróti, Z., \& Bereczki, C. (2020). Prevalence of hypertension in overweight and obese Hungarian children and adolescents. Orvosi hetilap, 161(4), 151-160.

Jeon, S. W, Chang, Y., Lim, S. W., Cho, J., Kim, H. N., Kim, K. B., \& Ryu, S. (2020). Associação bidirecional entre pressão arterial e sintomas depressivos em adultos jovens e de meia-idade: um estudo de coorte. Epidemiologia e ciências psiquiátricas, 29.

Kelishadi, R., Sadri, G., Tavasoli, A. A., Kahbazi, M., Roohafza, H. R., Sadeghi, M., \& Alikhassy, H. (2005). A prevalência cumulativa de fatores de risco para doença cardiovascular em adolescentes iranianos: IHHP-HHPC. Jornal de Pediatria, 81, 447-453.

Li, Y., Gu, H., Sinha, M. D., \& Chowienczyk, P. (2020). Hemodynamic characterization of primary hypertension in children and adolescents. Journal of the American Heart Association, 9(12), e015097. 
Research, Society and Development, v. 10, n. 11, e492101119949, 2021

(CC BY 4.0) | ISSN 2525-3409 | DOI: http://dx.doi.org/10.33448/rsd-v10i11.19949

Melnyk, BM e Fineout-Overholt, E. (Eds.). (2011). Prática baseada em evidências em enfermagem e saúde: um guia para as melhores práticas. Lippincott Williams \& Wilkins.

Organização Pan-America da Saúde. (2003). Doenças crónico-degenerativas e obesidade: estratégia mundial sobre alimentação saudável, actividade fisíca e saúde.

Pelazza, B. B., Gobbi, L. R. D. O. R., Puggina, A. C., Paula, C. R. D., Maia, L. G., \& Umpierrez, M. C. (2019). Adolescentes na fase pré-vestibular: um estudo da ansiedade, hipertensão, fatores antropométricos e hemodinâmicos associados. Nursing (Säo Paulo), 3398-3404.

Santos, A. M., Porelli, J. P., Jesus, K. E. M., \& Magalhães-Santos, I. F. (2018). Fatores de risco para hipertensão em jovens universitários. Revista de Ciências Médicas e Biológicas, 17(1), 52-60.

Siddiqui, S., \& Malatesta-Muncher, R. (2020). Hypertension in Children and Adolescents: A Review of Recent Guidelines. Pediatric Annals, 49(6), e250-e257.

Silva, F. A. C. C. D., Bragança, M. L. B. M., Bettiol, H., Cardoso, V. C., Barbieri, M. A. \& Silva, A. A. M. D. (2020). Status socioeconômico e fatores de risco cardiovascular em adultos jovens: uma análise transversal de uma coorte de nascimentos brasileira. Revista Brasileira de Epidemiologia, 23 , e200001.

Soares, A. F. A., Lima, L. F., de Almeida, B. B. A., de Santana Fukumitsu, M. K. M., \&amp; Motta, C. F. (2020). Prevalência da hipertensão arterial sistêmica em adolescentes na rede escolar pública de Valença-RJ. Revista Saber Digital, 13(1), 148-158.

Sousa, L. M. M., Marques-Vieira, C. M. A., Severino, S. S. P., \& Antunes, A. V. (2017). A metodologia de revisão integrativa da literatura em enfermagem. $N^{\circ} 21$ Série 2-Novembro 2017, 17.

Yusuf, S., Reddy, S., Ôunpuu, S., \& Anand, S. (2001). Carga global de doenças cardiovasculares: parte I: considerações gerais, a transição epidemiológica, fatores de risco e impacto da urbanização. Circulation, 104 (22), 2746-2753.

Wittkopf, P. G., Souza, C. A., Ferrari, E. P., Medeiros, T. E., \& Cardoso, F. L. (2016). Semelhanças entre comportamentos e papéis sociais apresentados por mulheres e homens com doença arterial coronariana. Arquivos de Ciências da Saúde, 23(2), 71-75.

Wang, H., Necheles, J., Carnethon, M., Wang, B., Li, Z., Wang, L., \& Wang, X. (2008). Medidas de adiposidade e pressão arterial em crianças e adolescentes chineses. Arquivos de doenças na infância, 93 (9), 738-744. 